\title{
Approximate controllability of a semilinear impulsive stochastic system with nonlocal conditions and Poisson jumps
}

\author{
A. Anguraj ${ }^{1 *}$, K. Ravikumar ${ }^{1}$ and Dumitru Baleanu ${ }^{2,3}$
}

\author{
"Correspondence: \\ angurajpsg@yahoo.com \\ 'Department of Mathematics, PSG \\ College of Arts and Science, \\ Coimbatore, India \\ Full list of author information is \\ available at the end of the article
}

\begin{abstract}
The objective of this paper is to investigate the approximate controllability of a semilinear impulsive stochastic system with nonlocal conditions and Poisson jumps in a Hilbert space. Nonlocal initial condition is a generalization of the classical initial condition and is motivated by physical phenomena. The results are obtained by using Sadovskii's fixed point theorem. Finally, an example is provided to illustrate the effectiveness of the obtained result.
\end{abstract}

MSC: $93 \mathrm{C} 25 ; 34 \mathrm{~K} 30 ; 34 \mathrm{~K} 35$

Keywords: Approximate controllability; Mild solutions; Impulsive systems; Poisson jumps

\section{Introduction}

The concept of controllability plays a major role in both finite and infinite dimensional spaces for systems represented by ordinary differential equations and partial differential equations. One of the basic qualitative behaviors of a dynamical system is the controllability. The problem of controllability is to show the existence of control function, which steers the solution of the system from its initial state to the final state, where the initial and final states may vary over the entire space. Conceived by Kalman, the controllability concept has been studied extensively in the fields of finite and infinite-dimensional systems. If a system cannot be controlled completely, then different types of controllability, such as approximate, null, local null, and local approximate null controllability, can be defined. For more details, the reader may refer to [1-9] and the references therein.

Meanwhile, the theory of impulsive differential equations describes processes which experience a sudden change of their state at certain moments. Dynamics of many evolutionary processes with such a characteristic arise naturally and are often, for example, population dynamics, control theory, physics, biology, medicine, etc. These perturbations can be well-approximated as instantaneous change of state or impulses. These processes are modeled by impulsive differential equations. Few works have been reported in the study of stochastic integrodifferential equations with impulsive effects, refer to [2, 10-13].

(c) The Author(s) 2020. This article is licensed under a Creative Commons Attribution 4.0 International License, which permits use sharing, adaptation, distribution and reproduction in any medium or format, as long as you give appropriate credit to the original author(s) and the source, provide a link to the Creative Commons licence, and indicate if changes were made. The images or other third party material in this article are included in the article's Creative Commons licence, unless indicated otherwise in a credit line to the material. If material is not included in the article's Creative Commons licence and your intended use is not permitted by statutory regulation or exceeds the permitted use, you will need to obtain permission directly from the copyright holder. To view a copy of this licence, visit http://creativecommons.org/licenses/by/4.0/ 
The Poisson jumps have become very popular, they are extensively used to model many of the phenomena arising in areas such as economics, finance, physics, biology, medicine, and other sciences. Moreover, many practical systems (such as sudden price variations [jumps] due to market crashes, earthquakes, epidemics, and so on) may undergo some jump-type stochastic perturbations. The sample paths of such systems are not continuous. Therefore, it is more appropriate to consider stochastic processes with jumps to describe such models. In general, these jump models are derived from Poisson random measure. The sample paths of such systems are right-continuous and have left limits. Recently, an increasing interest in the study of stochastic differential equations with jumps has been observed [14-16]. Luo and Liu [17] established the existence and uniqueness theory of mild solutions to stochastic partial functional differential equations with Markovian switching and Poisson jumps. It should be noted that most of the literature in this direction was mainly concerned with results on controllability of stochastic equations without jumps. However, up to now controllability problems for nonlinear stochastic dynamical systems with jumps have not been considered in the literature. In order to fill this gap, this paper considers the approximate controllability of semilinear stochastic control systems with nonlocal conditions using Sadovskii's fixed point theorem.

The goal of the present research work is to focus on studying the approximate controllability of a semilinear impulsive stochastic system with nonlocal conditions and Poisson jumps of the form

$$
\begin{aligned}
& d z(t)= {[A z(t)+B u(t)+f(t, z(t))] d t+\sigma(t, z(t)) d w(t) } \\
&+\int_{\mathcal{U}} h\left(t, z_{t}, u\right) \tilde{N}(d t, d u), \quad t \in J, t \neq t_{k}, \\
& \Delta z\left(t_{k}\right)= I_{k}\left(z\left(t_{k}\right)\right), \quad t=t_{k}, k=1,2, \ldots, m, \\
& z(0)=z_{0}+p(z),
\end{aligned}
$$

where $A$ is the infinitesimal generator of a semigroup of bounded linear operator $\{\mathcal{T}(t)\}$, $t \in[0, T]$ on $\mathrm{H}$. $B$ is a bounded linear operator from the Hilbert space $\mathrm{U}$ into $\mathrm{H}$. The control $u \in \mathscr{L}_{\mathcal{J}}^{2}([0, T], \mathrm{U}) ; f:[0, T] \times \mathrm{H} \rightarrow \mathrm{H} ; \sigma:[0, T] \times \mathrm{H} \rightarrow \mathscr{L}_{2}^{0} ; h:[0, T] \times \mathrm{H} \times \mathcal{U} \rightarrow \mathrm{H}$ and $I:$ $\mathrm{H} \rightarrow \mathrm{H}$ are suitable functions, and $p$ is a continuous function from $\mathscr{C}([0, T], \mathrm{H}) \rightarrow \mathrm{H}$. Also, the fixed moments of time $t_{k}$ satisfy $0=t_{0}<t_{1}<\cdots<t_{m}<t_{m+1}=b, z\left(t_{k}^{+}\right)$and $z\left(t_{k}^{-}\right)$denotes the right and left limits of $z(t)$ at $t=t_{k}$, respectively; $\Delta z\left(t_{k}\right)=z\left(t_{t}^{+}\right)-z\left(t_{k}^{-}\right)$represents the jump in the state $z$ at time $t_{k}$ with $I_{k}$ determining the size of the jump.

\section{Preliminaries}

Let $(\Omega, \Im, \mathbb{P})$ be a complete probability space with a normal filtration $\mathfrak{\Im}_{t}, t \in[0, T]$. Let $\mathrm{H}$, $\mathrm{U}$, and $\mathrm{E}$ be the separable Hilbert spaces. Let $w$ be a $Q$-Wiener process on $\left(\Omega, \mathfrak{\Im}_{T}, \mathbb{P}\right)$ with the covariance operator $Q$ such that $\operatorname{tr} Q<\infty$. Let us suppose that there exists a complete orthonormal system $e_{n}$ in E, a bounded sequence of real numbers $\lambda_{n}$, where $\lambda_{n}>0$ such that $Q e_{n}=\lambda_{n} e_{n}, n=1,2,3, \ldots$, and a sequence $\beta_{n}$ of independent Brownian motions such that

$$
w(t)=\sum_{n=1}^{\infty} \sqrt{\lambda_{n}} \beta_{n}(t) e_{n}, \quad t \in J,
$$


and $\mathfrak{\Im}_{t}=\mathfrak{\Im}_{t}^{\mathcal{W}}$, where $\mathfrak{\Im}_{t}^{\mathcal{W}}$ is the $\sigma$-algebra generated by $w$. Let $\mathscr{L}_{2}^{0}=\mathscr{L}_{2}\left(Q^{1 / 2} \mathrm{E} ; \mathrm{H}\right)$ be the space which consists of all Hilbert-Schmidt operators from $Q^{1 / 2} \mathrm{E}$ to $\mathrm{H}$ with the norm $\|\zeta\|=\operatorname{tr}\left[\zeta Q \zeta^{*}\right]$. Let $J_{1}=[-h, T]$ and the Banach space $\mathscr{P} \mathscr{C}\left(J_{1}, \mathscr{L}_{2}\left(\Omega, \Im_{t}, \mathrm{H}\right)\right)$ of all piecewise continuous functions $z(t)$ from $J_{1}=[-h, T]$ into $\mathscr{L}_{2}\left(\Omega, \Im_{t}, \mathrm{H}\right)$ be defined, which satisfies

$$
\sup _{t \in J_{1}} \mathbf{E}\|z(t)\|^{2}<\infty
$$

Let $\mathrm{H}_{2}$ be a closed subspace of $\mathscr{P} \mathscr{C}\left(J_{1}, \mathscr{L}_{2}\left(\Omega, \Im_{t}, \mathrm{H}\right)\right)$ that consists of all measurable and $\Im_{t}$-adapted processes $z(\cdot): t \in[-h, T]$ with the norm topology by

$$
\|\varphi\|_{\mathrm{H}_{2}}=\left(\sup _{t \in[0, b]} \mathbf{E}\|\varphi(t)\|_{\mathrm{H}}^{2}\right)^{1 / 2} \text {. }
$$

Suppose that $\{P(t), t \geq 0\}$ is a $\sigma$-finite stationary $\Im_{t}$-adapted Poisson point process taking values in measurable space $(\mathrm{U}, \mathscr{B}(\mathrm{U}))$. The random measure $N_{p}$ defined by $N_{p}((0, t] \times$ $\Lambda):=\sum_{s \in(0, t]} 1_{\Lambda}(P(s))$ for $\Lambda \in \mathscr{B}(\mathrm{U})$ is called the Poisson random measure induced by $P(\cdot)$, thus, we can define the measure $\tilde{N}$ by $\tilde{N}(d t, d u)=N_{\mathscr{P}}(d t, d u)-v(d u) d t$, where $v$ is the characteristic measure of $N_{P}$, which is called the compensated Poisson random measure.

Definition 2.1 ([2]) System (1) is approximately controllable on [0,T] if

$$
\overline{\mathrm{R}(T)}=\mathscr{L}_{2}\left(\Omega, \mathfrak{\Im}_{T}, \mathrm{H}\right)
$$

where $\mathrm{R}(T)=\left\{z(T ; u): u \in \mathscr{L}_{\Im}^{2}([0, T], \mathrm{U})\right\}$.

Definition 2.2 A stochastic process $z \in \mathrm{H}_{2}$ is a mild solution of (1) if, for each $u \in$ $\mathscr{L}_{\mathcal{S}}^{2}([0, T], \mathrm{U})$, it satisfies the integral equation

$$
\begin{aligned}
z(t)= & \mathcal{T}(t)\left[y_{0}+p(z)\right]+\int_{0}^{t} \mathcal{T}(t-s)[B u(s)+f(s, z(s))] d s \\
& +\int_{0}^{t} \mathcal{T}(t-s) \sigma(s, z(s)) d w(s)+\int_{0}^{t} \int_{\mathcal{U}} \mathcal{T}(t-s) h(s, z(s), u) \tilde{\mathrm{N}}(d s, d u) \\
& +\sum_{0<t_{k}<t} \mathcal{T}\left(t-t_{k}\right) I_{k}\left(z\left(t_{k}\right)\right) .
\end{aligned}
$$

The following are the main assumptions in this paper:

(H1) $T(t), t \geq 0$ is the strongly continuous semigroup of the bounded linear operator generated by $A$ such that $\max _{0 \leq t \leq T}\|T(t)\| \leq M$.

(H2) The functions $f:[0, T] \times \mathrm{H} \rightarrow \mathrm{H}$ and $\sigma:[0, T] \times \mathrm{H} \rightarrow \mathscr{L}_{2}^{0}$ satisfy that there exist $C_{1}>0, C_{2}>0, N_{1}>0$, and $N_{2}>0$ such that

$$
\begin{aligned}
& \left\|f\left(t, z_{1}\right)-f\left(t, z_{2}\right)\right\|^{2} \leq C_{1}\left\|z_{1}-z_{2}\right\|^{2}, \quad\left\|f\left(t, z_{1}\right)\right\|^{2} \leq C_{2}\left(1+\left\|z_{1}\right\|^{2}\right), \\
& \left\|\sigma\left(t, z_{1}\right)-\sigma\left(t, z_{2}\right)\right\|^{2} \leq N_{1}\left\|z_{1}-z_{2}\right\|^{2}, \quad\left\|\sigma\left(t, z_{1}\right)\right\|^{2} \leq N_{2}\left(1+\left\|z_{1}\right\|^{2}\right) \text {. }
\end{aligned}
$$

(H3) The function $h:[0, T] \times \mathrm{H} \times \mathcal{U} \rightarrow \mathrm{H}$ satisfies that there exist $K_{1}>0, K_{2}>0, L_{1}>0$, and $L_{2}>0$ such that

$$
\int_{\mathcal{U}}\left\|h\left(t, z_{1}, u\right)-h\left(t, z_{2}, u\right)\right\|_{\mathbb{X}}^{2} v(d u) \leq K_{1}\left\|z_{1}-z_{2}\right\|_{\mathbb{X}}^{2},
$$




$$
\begin{aligned}
& \int_{\mathcal{U}}\left\|h\left(t, z_{1}, u\right)\right\|_{\mathbb{X}}^{2} v(d u) \leq K_{2}\left(1+\left\|z_{1}\right\|_{\mathbb{X}}^{2}\right), \\
& \int_{\mathcal{U}}\left\|h\left(t, z_{1}, u\right)-h\left(t, z_{2}, u\right)\right\|_{\mathbb{X}}^{4} v(d u) \leq L_{1}\left\|z_{1}-z_{2}\right\|^{4}, \\
& \int_{\mathcal{U}}\left\|h\left(t, z_{1}, u\right)\right\|^{4} v(d u) \leq L_{2}\left(1+\left\|z_{1}\right\|^{4}\right) .
\end{aligned}
$$

(H4) There exist some positive constants $M_{p}$ such that

$$
\left\|p\left(z_{1}\right)-p\left(z_{2}\right)\right\|^{2} \leq M_{p}\left\|z_{1}-z_{2}\right\|^{2}, \quad\left\|p\left(z_{1}\right)\right\|^{2} \leq M_{p}\left(1+\left\|z_{1}\right\|^{2}\right) .
$$

(H5) $I_{k}: \mathrm{H} \rightarrow \mathrm{H}$ satisfies

$$
\left\|I_{k}\left(z_{1}\right)-I_{k}\left(z_{2}\right)\right\|^{2} \leq q_{k}\left\|z_{1}-z_{2}\right\|^{2}, \quad\left\|I_{k}\left(z_{1}\right)\right\|^{2} \leq q_{k}\left(1+\left\|z_{1}\right\|^{2}\right) .
$$

(H6) For each $0 \leq t<T$, the operator $\zeta\left(\zeta I+\Gamma_{t}^{T}\right)^{-1} \rightarrow 0$ is the strong operator topology as $\zeta \rightarrow 0^{+}$, where

$$
\Gamma_{t}^{T}=\int_{t}^{T} \mathcal{T}(T-s) B B^{*} \mathcal{T}^{*}(T-s) d s
$$

is the controllability Gramian. Observe that the linear deterministic system corresponding to (1)

$$
\begin{aligned}
& d z(t)=[A z(t)+B u(t)] d t, \quad t \in[0, T], \\
& z(0)=z_{0},
\end{aligned}
$$

is approximately controllable on $[t, T]$ iff the operator $\zeta\left(\zeta I+\Gamma_{t}^{T}\right)^{-1} \rightarrow 0$ strongly as $\zeta \rightarrow 0^{+}$.

\section{Main result}

Lemma 3.1 ([18]) For any $z_{T} \in \mathscr{L}_{2}\left(\Omega, \Im_{T}, \mathrm{H}\right)$, there exists $\phi \in \mathscr{L}_{2}^{\Im}\left([0, T], \mathscr{L}_{2}^{0}\right)$ such that $z_{T}=\mathbf{E} z_{T}+\int_{0}^{T} \phi(s) d w(s)$.

For any $\zeta>0$ and $z_{T} \in \mathscr{L}_{2}\left(\Omega, \Im_{T}, \mathrm{H}\right)$, we define the control function

$$
\begin{aligned}
u^{\zeta}\left(t, z_{1}\right) & \\
= & B^{*} \mathcal{T}^{*}(T-t)\left[\left(\zeta I+\Psi_{0}^{T}\right)^{-1}\left(\mathbf{E} z_{T}-\mathcal{T}(T)\left(z_{0}+h\left(z_{1}\right)\right)\right)+\int_{0}^{t}\left(\zeta I+\Psi_{s}^{T}\right)^{-1} \phi(s) d w(s)\right] \\
& -B^{*} \mathcal{T}^{*}(T-t) \int_{0}^{t}\left(\zeta I+\Psi_{s}^{T}\right)^{-1} \mathcal{T}(T-s) f\left(s, z_{1}(s)\right) d s \\
& -B^{*} \mathcal{T}^{*}(T-t) \int_{0}^{t}\left(\zeta I+\Psi_{s}^{T}\right)^{-1} \mathcal{T}(T-s) \sigma\left(s, z_{1}(s)\right) d w(s) \\
& -B^{*} \mathcal{T}^{*}(T-t) \int_{0}^{t} \int_{\mathcal{U}}\left(\zeta I+\Psi_{s}^{T}\right)^{-1} \mathcal{T}(T-s) h\left(s, z_{1}(s), u\right) \tilde{\mathrm{N}}(d s, d u) \\
& -B^{*} \mathcal{T}^{*}(T-t)\left(\zeta I+\Psi_{s}^{T}\right)^{-1} \sum_{k=1}^{m} \mathcal{T}\left(T-t_{k}\right) I_{k}\left(z_{1}\left(t_{k}\right)\right) .
\end{aligned}
$$


Lemma 3.2 There exists $\mathrm{M}_{u}>0$ such that, for all $z_{1}, z_{2} \in \mathrm{H}_{2}$, we have

$$
\begin{aligned}
& \mathbf{E}\left\|u^{\zeta}\left(t, z_{1}\right)-u^{a}\left(t, z_{2}\right)\right\|^{2} \leq \frac{\mathrm{M}_{u}}{\zeta^{2}}\left\|z_{1}-z_{2}\right\|^{2}, \\
& \mathbf{E}\left\|u^{\zeta}\left(t, z_{1}\right)\right\|^{2} \leq \frac{\mathrm{M}_{u}}{\zeta^{2}}\left(1+\left\|z_{1}\right\|^{2}\right) .
\end{aligned}
$$

Proof Let $z_{1}, z_{2} \in \mathrm{H}_{2}$. By hypothesis and Holder's inequality, we obtain

$$
\begin{aligned}
& \mathbb{E}\left\|u^{\zeta}\left(t, z_{1}\right)-u^{\zeta}\left(t, z_{2}\right)\right\|^{2} \\
& \leq 5 \mathbf{E}\left\|B^{*} \mathcal{T}^{*}(T-t)\left(\zeta I+\Psi_{0}^{T}\right)^{-1} \mathcal{T}(T)\left[p\left(z_{1}\right)-p\left(z_{2}\right)\right]\right\|^{2} \\
& +5 \mathbf{E}\left\|B^{*} \mathcal{T}^{*}(T-t) \int_{0}^{t}\left(\zeta I+\Psi_{s}^{T}\right)^{-1} \mathcal{T}(T-s)\left[f\left(s, z_{1}(s)\right)-f\left(s, z_{2}(s)\right)\right] d s\right\|^{2} \\
& +5 \mathbf{E}\left\|B^{*} \mathcal{T}^{*}(T-t) \int_{0}^{t}\left(\zeta I+\Psi_{s}^{T}\right)^{-1} \mathcal{T}(T-s)\left[\sigma\left(s, z_{1}(s)\right)-\sigma\left(s, z_{2}(s)\right)\right] d w(s)\right\|^{2} \\
& +5 \mathbf{E} \| B^{*} \mathcal{T}^{*}(T-t) \int_{0}^{t} \int_{\mathcal{U}}\left(\zeta I+\Psi_{s}^{T}\right)^{-1} \mathcal{T}(T-s)\left[h\left(s, z_{1}(s), u\right)-h\left(s, z_{2}(s), u\right)\right] \\
& \times \tilde{\mathrm{N}}(d s, d u) \|^{2} \\
& +5 \mathbf{E}\left\|B^{*} \mathcal{T}^{*}(T-t)\left(\zeta I+\Psi_{s}^{T}\right)^{-1} \sum_{k=1}^{m} \mathcal{T}\left(T-t_{k}\right)\left[I_{k}\left(z_{1}\left(t_{k}\right)\right)-I_{k}\left(z_{2}\left(t_{k}\right)\right)\right]\right\|^{2} \\
& \leq \frac{5}{\zeta^{2}} M_{B}^{2} M^{4} M_{g}\left\|z_{1}-z_{2}\right\|_{\mathrm{H}}^{2}+\frac{5}{\zeta^{2}} M_{B}^{2} M^{4} b \int_{0}^{t} C_{1} \mathbf{E}\left\|z_{1}(s)-z_{2}(s)\right\|_{\mathrm{H}}^{2} d s \\
& +\frac{5}{\zeta^{2}} M_{B}^{2} M^{4} L_{\sigma} \int_{0}^{t} N_{1} \mathbf{E}\left\|z_{1}(s)-z_{2}(s)\right\|_{\mathrm{H}}^{2} d s \\
& \leq \frac{5}{\zeta^{2}} M_{B}^{2} M^{4} M_{g}\left\|z_{1}-z_{2}\right\|_{\mathrm{H}}^{2}+\frac{5}{\zeta^{2}} M_{B}^{2} M^{4} b \int_{0}^{t} C_{1} \mathbf{E}\left\|z_{1}(s)-z_{2}(s)\right\|_{\mathrm{H}}^{2} d s \\
& +\frac{5}{\zeta^{2}} M_{B}^{2} M^{4} L_{\sigma} \int_{0}^{t} N_{1} \mathbf{E}\left\|z_{1}(s)-z_{2}(s)\right\|_{\mathrm{H}}^{2} d s \\
& +\frac{5}{\zeta^{2}} M_{B}^{2} M^{4} \int_{0}^{t} \int_{\mathcal{U}} \mathbf{E}\left\|h\left(s, z_{1}(s), u\right)-h\left(s, z_{2}(s), u\right)\right\|_{\mathrm{H}}^{2} v(d u) d s \\
& +\frac{5}{\zeta^{2}} M_{B}^{2} M^{4}\left(\int_{0}^{t} \int_{\mathcal{U}} \mathbf{E}\left\|h\left(s, z_{1}(s), u\right)-h\left(s, z_{2}(s), u\right)\right\|^{4} v(d u) d s\right)^{1 / 2} \\
& +\frac{5}{\zeta^{2}} M_{B}^{2} M^{4} K \int_{0}^{t} \mathbf{E}\left\|z_{1}-z_{2}\right\|_{\mathrm{H}}^{2} d s \\
& \leq \frac{5}{\zeta^{2}} M_{B}^{2} M^{4}\left[M_{p}+C_{1} T^{2}+L_{\sigma} N_{1} T+K_{1} b+\sqrt{L_{1}} \sqrt{T}+K\right]\left\|z_{1}-z_{2}\right\|_{\mathrm{H}_{2}}^{2} \\
& =\frac{\mathrm{M}_{u}}{\zeta^{2}}\left\|z_{1}-z_{2}\right\|_{\mathrm{H}_{2}}^{2} \text {, }
\end{aligned}
$$

where $K=\mathbf{E} \sum_{k=1}^{m}\left\|q_{k}\right\|^{2}, \mathrm{M}_{u}=5 M_{B}^{2} M^{4}\left[M_{p}+C_{1} T^{2}+L_{G} N_{1} T+K_{1} T+\sqrt{L_{1}} \sqrt{T}+K\right]$. When $u^{\zeta}\left(t, z_{2}\right)=0$, the second inequality can be proved in the same approach. 
Theorem 3.1 Assume that (H1)-(H5) are satisfied. Then equations (1) have mild solution on $[0, T]$ with the result that

$$
\begin{aligned}
& 12 M^{2} M_{p}+6 M^{2}\left(6 M_{B}^{2} T^{2} \frac{\mathrm{M}_{u}}{\zeta^{2}}+T^{2} C_{2}+L_{G} N_{2} T+T K_{2}+\sqrt{L_{2}} \sqrt{T}+K\right)<1 \\
& 5 M^{2} M_{B}^{2} T \frac{\mathrm{M}_{u}}{\zeta^{2}}+5 M^{2} T C_{1}+5 M^{2} L_{G} N_{1} T+5 M^{2} K_{1} T+5 M^{2} \sqrt{L_{1}} \sqrt{T}+5 M^{2} K T<1
\end{aligned}
$$

Proof Define the operator $\Theta_{\zeta}: \mathrm{H}_{2} \rightarrow \mathrm{H}_{2}$, for $\zeta>0$, by

$$
\begin{aligned}
\left(\Theta_{\zeta} z\right)(t)= & T(t)\left[z_{0}+p(z)\right]+\int_{0}^{t} \mathcal{T}(t-s)\left[B u^{\zeta}(s, z)+f(s, z(s))\right] d s \\
& +\int_{0}^{t} \mathcal{T}(t-s) \sigma(s, z(s)) d w(s)+\int_{0}^{t} \int_{\mathcal{U}} \mathcal{T}(t-s) h(s, z(s), u) \tilde{\mathrm{N}}(d s, d u) \\
& +\sum_{0<t_{k}<t} \mathcal{T}\left(t-t_{k}\right) I_{k}\left(z\left(t_{k}\right)\right) .
\end{aligned}
$$

Step 1 . For any $z \in \mathrm{H}_{2}, \Theta_{a}(z)(t)$ is continuous on $[0, T]$.

By assumptions and Holder's inequality, we have

$$
\begin{aligned}
& \mathbf{E}\left\|\left(\Theta_{\zeta} z\right)\left(t_{2}\right)-\left(\Theta_{\zeta} z\right)\left(t_{1}\right)\right\|^{2} \\
& \leq 11\left[\left[2\left(\mathbf{E}\left\|\left(\mathcal{T}\left(t_{2}\right)-\mathcal{T}\left(t_{1}\right)\right) z_{0}\right\|^{2}+\mathbf{E}\left\|\left(\mathcal{T}\left(t_{2}\right)-\mathcal{T}\left(t_{1}\right)\right) p(z)\right\|^{2}\right)\right]\right. \\
& +t_{1} \int_{0}^{t_{1}} \mathbf{E}\left\|\left[\mathcal{T}\left(t_{2}-s\right)-\mathcal{T}\left(t_{1}-s\right)\right] f(s, z(s))\right\|^{2} d s \\
& +M^{2}\left(t_{2}-t_{1}\right) \int_{t_{1}}^{t_{2}} \mathbf{E}\|f(s, z(s))\|^{2} d s \\
& +L_{\sigma} \int_{0}^{t_{1}} \mathbf{E}\left\|\left[\mathcal{T}\left(t_{2}-s\right)-\mathcal{T}\left(t_{1}-s\right)\right] \sigma(s, z(s))\right\|^{2} d s \\
& +M^{2} L_{\sigma} \int_{t_{1}}^{t_{2}} \mathbf{E}\|\sigma(s, z(s))\|^{2} d s \\
& +t_{1} \int_{0}^{t_{1}} \mathbf{E}\left\|\left[\mathcal{T}\left(t_{2}-s\right)-\mathcal{T}\left(t_{1}-s\right)\right] B u^{\zeta}(s, z)\right\|^{2} d s \\
& +\|B\|^{2} M^{2}\left(t_{2}-t_{1}\right) \int_{t_{1}}^{t_{2}} \mathbf{E}\left\|u^{\zeta}(s, z)\right\|^{2} d s \\
& +\int_{0}^{t_{1}} \int_{\mathcal{U}} \mathbf{E}\left\|\left[\mathcal{T}\left(t_{2}-s\right)-\mathcal{T}\left(t_{1}-s\right)\right] h(s, z(s), u)\right\|^{2} v(d u) d s \\
& +\left(\int_{0}^{t_{1}} \int_{\mathbb{U}} \mathbf{E}\left\|\left[\mathcal{T}\left(t_{2}-s\right)-\mathcal{T}\left(t_{1}-s\right)\right] h(s, z(s), u)\right\|^{4} v(d u) d s\right)^{1 / 2} \\
& +\int_{t_{1}}^{t_{2}} \int_{\mathcal{U}} \mathbf{E}\left\|\mathcal{T}\left(t_{2}-s\right) h(s, z(s), u)\right\|^{2} v(d u) d s \\
& +\left(\int_{t_{1}}^{t_{2}} \int_{\mathcal{U}} \mathbf{E}\left\|\mathcal{T}\left(t_{2}-s\right) h(s, z(s), u)\right\|^{4} v(d u) d s\right)^{1 / 2}
\end{aligned}
$$




$$
\begin{aligned}
& +\sum_{0<t_{k}<t_{1}} \mathbf{E}\left(\left\|\mathcal{T}\left(t_{2}-t_{k}\right)-\mathcal{T}\left(t_{1}-t_{k}\right)\right\|\left\|I_{k}\left(z\left(t_{k}\right)\right)\right\|\right)^{2} \\
& \left.+\sum_{t_{1}<t_{k}<t_{2}} \mathbf{E}\left(\left\|\mathcal{T}\left(t_{2}-t_{k}\right)\right\|\left\|I_{k}\left(z\left(t_{k}\right)\right)\right\|\right)^{2}\right] .
\end{aligned}
$$

Thus utilizing LDC theorem, the RHS of the considered inequality tends to zero since $\left(t_{2}-t_{1}\right) \rightarrow 0$. Accordingly, $\Theta_{\zeta}(z)(t)$ is continuous from the right in [0,T). A relative intent demonstrates that $\Theta_{\zeta}(z)(t)$ is similarly continuous from the left in $(0, T]$. Consequently, on $[0, T], \Theta_{\zeta}(z)(t)$ is continuous.

Step 2. Let $B_{n}=\left\{z \in \mathrm{H}_{2}: \mathbf{E}\|z(t)\|_{\mathrm{H}}^{2} \leq n\right\}$, then the set $B_{n}$ is obviously a bounded, closed, and convex set in $\mathrm{H}_{2}$ for each integer $n>0$.

From Holder's inequality and (H1), we get

$$
\begin{aligned}
\mathbf{E}\left\|\int_{0}^{t} \mathcal{T}(t-s) f(s, z(s)) d s\right\|_{\mathrm{H}}^{2} & \leq \mathbf{E}\left[\int_{0}^{t}\|\mathcal{T}(t-s) f(s, z(s))\|_{\mathrm{H}} d s\right]^{2} \\
& \leq M^{2} T \int_{0}^{t} C_{2}\left(1+\mathbf{E}\|z(s)\|_{\mathrm{H}}^{2}\right) d s \\
& \leq M^{2} T C_{2} \int_{0}^{t}\left(1+\sup _{s \in[0, T]} \mathbf{E}\|z(s)\|_{\mathrm{H}}^{2}\right) d s \\
& \leq M^{2} T^{2} C_{2}\left(1+\|z\|_{\mathrm{H}}^{2}\right) .
\end{aligned}
$$

From $(\mathrm{H} 2)$, we get

$$
\begin{aligned}
\mathbf{E}\|\mathcal{T}(t-s) \sigma(s, z(s)) d w(s)\| & \leq L_{\sigma} \int_{0}^{t} \mathbf{E}\|\mathcal{T}(t-s) \sigma(s, z(s))\|_{L_{2}^{0}}^{2} d s \\
& \leq L_{\sigma} M^{2} N_{2} \int_{0}^{t}\left(1+\sup _{s \in[0, b]} \mathbf{E}\|z(s)\|_{\mathbb{H}}^{2}\right) d s \\
& \leq L_{\sigma} M^{2} N_{2} T\left(1+\|z\|_{\mathbb{H}_{2}}^{2}\right) .
\end{aligned}
$$

Next, from (H4) and (H5), we get

$$
\begin{aligned}
\mathbf{E}\left\|\int_{0}^{t} \int_{\mathcal{U}} \mathcal{T}(t-s) h(s, z(s), u) \tilde{\mathrm{N}}(d s, d u)\right\|^{2} \leq & M^{2}\left(\int_{0}^{t} \int_{\mathcal{U}} \mathbf{E}\|h(s, z(s), u)\|^{2} v(d u) d s\right) \\
& +M^{2}\left(\int_{0}^{t} \int_{\mathcal{U}} \mathbf{E}\|h(s, z(s), u)\|^{4} v(d u) d s\right)^{1 / 2} \\
\leq & M^{2} T K_{2}\left(1+\|z\|_{\mathrm{H}}^{2}\right)+M^{2} \sqrt{L_{2}} \sqrt{T}\left(1+\|z\|_{\mathrm{H}}^{2}\right) .
\end{aligned}
$$

Similarly, from assumption (H7), we get

$$
\mathbf{E}\left\|\sum_{0<t_{k}<t} \mathcal{T}\left(t-t_{k}\right) I_{k}\left(z\left(t_{k}\right)\right)\right\|^{2} \leq M^{2} K\left(1+\|z\|_{\mathrm{H}}^{2}\right) .
$$

Now, we have to prove that there exists a number $n>0$ such that $\Theta_{\zeta}\left(B_{n}\right) \subseteq B_{n}$. 
If not, for each $n>0$, there exists a function $z_{n}(\cdot) \in B_{n}$ but $\Theta_{\zeta} z_{n}$ does not belong to $B_{n}$, that is, $\mathbf{E}\left\|\Theta_{\zeta} z_{n}(t)\right\|_{\mathrm{H}}^{2}>n$ for $t \in J$. Also, by Lemma 3.2 and hypotheses (H2), (H3), we get

$$
\begin{aligned}
n \leq & \mathbf{E}\left\|\Theta_{\zeta} z_{n}(t)\right\|_{\mathrm{H}}^{2} \\
= & 6 \mathbf{E}\left\|\mathcal{T}(t)\left[z_{0}+p(z)\right]\right\|_{\mathrm{H}}^{2}+6 \mathbf{E}\left\|\int_{0}^{t} \mathcal{T}(t-s) B u^{\zeta}(s, z)\right\|_{\mathrm{H}}^{2} \\
& +6 \mathbf{E}\left\|\int_{0}^{t} \mathcal{T}(t-s) f(s, z(s)) d s\right\|_{\mathrm{H}}^{2}+6 \mathbf{E}\left\|\int_{0}^{t} \mathcal{T}(t-s) \sigma(s, z(s)) d w(s)\right\|_{\mathrm{H}}^{2} \\
& +6 \mathbf{E}\left\|\int_{0}^{t} \int_{\mathcal{U}} \mathcal{T}(t-s) h(s, z(s), u) \tilde{\mathrm{N}}(d s, d u)\right\|_{\mathrm{H}}^{2}+6 \mathbf{E}\left\|\sum_{0<t_{k}<t} \mathcal{T}\left(t-t_{k}\right) I_{k}\left(z\left(t_{k}\right)\right)\right\|_{\mathrm{H}}^{2} \\
\leq & 6 M^{2}\left[2 \mathbf{E}\left\|z_{0}\right\|^{2}+2 \mathbf{E}\|p(z)\|^{2}\right]+6 M^{2} M_{B}^{2} T^{2} \frac{\mathrm{M}_{u}}{\zeta^{2}}\left(1+\|z\|_{\mathrm{H}}^{2}\right) \\
& +6 M^{2} T^{2} C_{2}\left(1+\|z\|_{\mathrm{H}}^{2}\right)+6 L_{\sigma} M^{2} N_{2} T\left(1+\|z\|_{\mathrm{H}}^{2}\right)+6 M^{2} T K_{2}\left(1+\|z\|_{H}^{2}\right) \\
& +6 M^{2} \sqrt{L_{2}} \sqrt{T}\left(1+\|z\|_{\mathrm{H}}^{2}\right)+6 M^{2} K\left(1+\|z\|_{\mathrm{H}}^{2}\right) \\
\leq & 12 M^{2} \mathbf{E}\left\|z_{0}\right\|^{2}+12 M^{2} M_{p}(1+n)+6 M^{2} M_{B}^{2} T^{2} \frac{\mathrm{M}_{u}}{\zeta^{2}}(1+n) \\
& +6 M^{2} b^{2} C_{2}(1+n)+6 L_{G} M^{2} N_{2} T(1+n)+6 M^{2} T K_{2}(1+n) \\
& +6 M^{2} \sqrt{L_{2}} \sqrt{b}(1+n)+6 M^{2} K(1+n) \\
\leq & \left(12 M^{2} \mathbf{E}\left\|z_{0}\right\|^{2}+12 M^{2} M_{p}+6 M^{2} M_{B}^{2} T^{2} \frac{\mathrm{M}_{u}}{a^{2}}+6 M^{2} T^{2} C_{2}\right. \\
& \left.+6 L_{G} M^{2} N_{2} T+6 M^{2} T K_{2}+6 M^{2} \sqrt{L_{2}} \sqrt{T T}+6 M^{2} K\right) \\
& +\left(12 M^{2} M_{p}+6 M^{2} M_{B}^{2} T^{2} \frac{\mathrm{M}_{u}}{a^{2}}+6 M^{2} T^{2} C_{2}\right. \\
& \left.+6 L_{G} M^{2} N_{2} T+6 M^{2} T K_{2}+6 M^{2} \sqrt{L_{2}} \sqrt{b}+6 M^{2} K\right) n . \\
&
\end{aligned}
$$

Now dividing each side by $n$ and considering the limit as $n \rightarrow \infty$, we get

$$
12 M^{2} M_{p}+6 M^{2}\left(6 M_{B}^{2} T^{2} \frac{\mathrm{M}_{u}}{\zeta^{2}}+T^{2} C_{2}+L_{\sigma} N_{2} T+T K_{2}+\sqrt{L_{2}} \sqrt{T}+K\right)>1 .
$$

This is a contradiction to condition (5). Thus, $\Theta_{\zeta} B_{n} \subseteq B_{n}$ for any $n>0$.

Step 3. The operators $\Theta_{\zeta_{1}}$ and $\Theta_{\zeta_{2}}$ are defined as follows:

$$
\begin{aligned}
\left(\Theta_{\zeta_{1}} z\right)(t)= & \mathcal{T}(t)\left[z_{0}+p(z)\right] \\
\left(\Theta_{\zeta_{2}} z\right)(t)= & \int_{0}^{t} \mathcal{T}(t-s)\left[B u^{\zeta}(s, z)+f(s, z(s))\right] d s+\int_{0}^{t} \mathcal{T}(t-s) \sigma(s, z(s)) d w(s) \\
& +\int_{0}^{t} \int_{\mathcal{U}} \mathcal{T}(t-s) h(s, z(s), u) \tilde{\mathrm{N}}(d s, d u)+\sum_{0<t_{k}<t} \mathcal{T}\left(t-t_{k}\right) I_{k}\left(z\left(t_{k}\right)\right), \quad t \in[0, T] .
\end{aligned}
$$


From (H3) it is clear that $\Theta_{\zeta_{1}}$ is completely continuous, then for $z_{1}, z_{2} \in B_{n}$. Hence from hypotheses $(\mathrm{H} 2),(\mathrm{H} 3)$ and for any $t \in[0, T]$, we get

$$
\begin{aligned}
& \mathbf{E}\left\|\left(\Theta_{\zeta_{2}} z_{1}\right)(t)-\left(\Theta_{\zeta_{2}} z_{2}\right)(t)\right\|_{\mathrm{H}}^{2} \\
& \leq 5 \mathbf{E}\left\|\int_{0}^{t} \mathcal{T}(t-s) B\left[u^{\zeta}\left(s, z_{1}\right)-u^{\zeta}\left(s, z_{2}\right)\right] d s\right\|_{\mathrm{H}}^{2} \\
&+5 \mathbf{E}\left\|\int_{0}^{t} \mathcal{T}(t-s)\left[f\left(s, z_{1}(s)\right)-f\left(s, z_{2}(s)\right)\right] d s\right\|_{\mathrm{H}}^{2} \\
&+5 \mathbf{E}\left\|\int_{0}^{t} \mathcal{T}(t-s)\left[\sigma\left(s, z_{1}(s)\right)-\sigma\left(s, z_{2}(s)\right)\right] d w(s)\right\|_{\mathrm{H}}^{2} \\
&+5 \mathbf{E}\left\|\int_{0}^{t} \mathcal{T}(t-s) \int_{\mathcal{U}}\left[h\left(s, z_{1}(s), u\right)-h\left(s, z_{2}(s), u\right)\right] \tilde{\mathrm{N}}(d s, d u)\right\|_{\mathrm{H}}^{2} \\
&+5 \mathbf{E}\left\|\sum_{0<t_{k}<t} \mathcal{T}\left(t-t_{k}\right) I_{k}\left[\left(z_{1}\left(t_{k}\right)-z_{2}\left(t_{k}\right)\right)\right]\right\|_{\mathrm{H}}^{2} \\
& \leq 5 M^{2} M_{B}^{2} b \frac{\mathrm{M}_{u}}{\zeta^{2}}\left\|z_{1}-z_{2}\right\|_{\mathrm{H}}^{2}+5 M^{2} T C_{1}\left\|z_{1}-z_{2}\right\|_{\mathrm{H}}^{2}+5 M^{2} L_{\sigma} N_{1} T\left\|z_{1}-z_{2}\right\|_{\mathrm{H}}^{2} \\
&+5 M^{2} K_{1} T\left\|z_{1}-z_{2}\right\|_{\mathrm{H}}^{2}+5 M^{2} \sqrt{L_{1}} \sqrt{T}\left\|z_{1}-z_{2}\right\|_{\mathrm{H}}^{2}+5 M^{2} K T\left\|z_{1}-z_{2}\right\|_{\mathrm{H}}^{2} . \\
& \leq\left(5 M^{2} M_{B}^{2} T \frac{\mathrm{M}_{u}}{\zeta^{2}}+5 M^{2} T C_{1}+5 M^{2} L_{\sigma} N_{1} T\right. \\
&\left.+5 M^{2} K_{1} T+5 M^{2} \sqrt{L_{1}} \sqrt{T}+5 M^{2} K T\right)\left\|z_{1}-z_{2}\right\|_{\mathrm{H}}^{2} .
\end{aligned}
$$

Therefore

$$
\mathbf{E}\left\|\left(\Theta_{\zeta_{2}} z_{1}\right)(t)-\left(\Theta_{\zeta_{2}} z_{2}\right)(t)\right\|_{\mathrm{H}}^{2} \leq L_{0}\left\|z_{1}-z_{2}\right\|_{\mathrm{H}}^{2},
$$

where

$$
\begin{aligned}
L_{0} & =\left(5 M^{2} M_{B}^{2} T \frac{\mathrm{M}_{u}}{a^{2}}+5 M^{2} T C_{1}+5 M^{2} L_{\sigma} N_{1} T+5 M^{2} K_{1} T+5 M^{2} \sqrt{L_{1}} \sqrt{T}+5 M^{2} K T\right) \\
& <1 .
\end{aligned}
$$

Thus $\Theta_{\zeta_{2}}$ is a contraction mapping.

Now we have a condensing mapping $\Theta_{\zeta}=\Theta_{\zeta_{1}}+\Theta_{\zeta_{2}}$ on $B_{n}$. Thus, by Sadovskii's fixed point theorem, the mild solution of (1) is the fixed point $z(\cdot)$ for $\Theta_{\zeta}$ on $B_{n}$.

Theorem 3.2 Assume that (H1)-(H5) hold and iff, $\sigma$, and h are uniformly bounded, then equation (1) is approximately controllable on $[0, T]$.

Proof Let $z_{a}$ be a fixed point of $\Theta_{\zeta}$ in $\mathrm{H}_{2}$. By using stochastic Fubini's theorem, we can clearly see that

$$
\begin{aligned}
z_{\zeta}(T)= & z_{T}-\zeta\left(\zeta I+\Gamma_{0}^{T}\right)^{-1}\left(\mathbf{E} z_{T}-\mathcal{T}(T)\left(z_{0}+p(z)\right)\right) \\
& +\zeta \int_{0}^{T}\left(\zeta I+\Gamma_{s}^{T}\right)^{-1} \mathcal{T}(b-s) f\left(s, z_{a}(s)\right) d s
\end{aligned}
$$




$$
\begin{aligned}
& +\zeta \int_{0}^{T}\left(\zeta I+\Gamma_{s}^{T}\right)^{-1}\left[\mathcal{T}(T-s) \sigma\left(s, z_{a}(s)\right)-\phi(s)\right] d w(s) \\
& +\zeta \int_{0}^{T} \int_{\mathcal{U}}\left(a I+\Gamma_{s}^{T}\right)^{-1}\left[\mathcal{T}(T-s) h\left(s, z_{a}(s), u\right) \tilde{\mathrm{N}}(d s, d u)\right] \\
& +\zeta\left(\zeta I+\Gamma_{s}^{T}\right)^{-1} \sum_{0<t_{k}<t} \mathcal{T}\left(T-t_{k}\right) I_{k}\left(z_{a}\left(t_{k}\right)\right) .
\end{aligned}
$$

From our assumptions, $f, \sigma$, and $h$ are uniformly bounded, then there exists $\mathrm{D}>0$ such that

$$
\left\|f\left(s, z_{a}(s)\right)\right\|^{2}+\left\|\sigma\left(s, z_{a}(s)\right)\right\|^{2}+\left\|h\left(s, z_{a}(s), u\right)\right\|^{2} \leq \mathrm{D}
$$

in $[0, T] \times \Omega$. Now there exist subsequences, indicated by $\left\{f\left(s, z_{a}(s)\right)\right\},\left\{\sigma\left(s, z_{a}(s)\right)\right\}$, and $\left\{h\left(s, z_{a}(s), u\right)\right\}$, which converge weakly to $f(s), \sigma(s)$ in $\mathrm{H} \times L_{2}^{0}$ and $h(s, u)$ in $\mathrm{H} \times \mathrm{H} \times L_{2}^{0}$ respectively. Thus, the compactness of $\mathcal{T}(t)$ gives $\mathcal{T}(T-s) f\left(s, z_{a}(s)\right) \rightarrow \mathcal{T}(T-s) f(s)$, $\mathcal{T}(T-s) \sigma\left(s, z_{a}(s)\right) \rightarrow \mathcal{T}(T-s) \sigma(s), \mathcal{T}(T-s) h\left(s, z_{a}(s), u\right) \rightarrow \mathcal{T}(T-s) h(s, u)$ in $[0, T] \times \Omega$. Then we have

$$
\begin{aligned}
\mathbf{E} \| z_{\zeta}(T) & -z_{T} \|^{2} \\
\leq & 9 \mathbf{E}\left\|\zeta\left(\zeta I+\Gamma_{0}^{T}\right)^{-1}\left[\mathbf{E} z_{T}-\mathcal{T}(T)\left[z_{0}+p(z)\right]\right]\right\|^{2} \\
& +9 \mathbf{E}\left(\int_{0}^{T}\left\|\zeta\left(\zeta I+\Gamma_{0}^{T}\right)^{-1} \phi(s)\right\|_{\mathscr{L}_{2}^{0}}^{2} d s\right) \\
& +9 \mathbf{E}\left(\int_{0}^{T}\left\|\zeta\left(\zeta I+\Gamma_{s}^{T}\right)^{-1}\right\|\left\|\mathcal{T}(T-s)\left[f\left(s, z_{a}(s)\right)-f(s)\right]\right\| d s\right)^{2} \\
& +9 \mathbf{E}\left(\int_{0}^{T}\left\|\zeta\left(\zeta I+\Gamma_{s}^{T}\right)^{-1} \mathcal{T}(T-s) f(s)\right\| d s\right)^{2} \\
& +9 \mathbf{E}\left(\int_{0}^{T}\left\|\zeta\left(\zeta I+\Gamma_{s}^{T}\right)^{-1}\right\|\left\|\mathcal{T}(T-s)\left[\sigma\left(s, z_{a}(s)\right)-\sigma(s)\right]\right\|_{\mathscr{L}_{2}^{0}}^{2} d s\right) \\
& +9 \mathbf{E}\left(\int_{0}^{T}\left\|\zeta\left(\zeta I+\Gamma_{s}^{T}\right)^{-1} \mathcal{T}(T-s) \sigma(s)\right\|_{\mathscr{L}_{2}^{0}}^{2} d s\right) \\
& +9 \mathbf{E}\left(\int_{0}^{T} \int_{\mathcal{U}}\left\|\zeta\left(\zeta I+\Gamma_{s}^{T}\right)^{-1}\right\|\left\|\mathcal{T}(T-s)\left[h\left(s, z_{a}(s)\right)-h(s, u)\right] \tilde{\mathrm{N}}(d s, d u)\right\| d s\right)^{2} \\
& +9 \mathbf{E}\left(\int_{0}^{T}\left\|\zeta\left(\zeta I+\Gamma_{s}^{T}\right)^{-1} \mathcal{T}(T-s) h(s, u) \tilde{\mathrm{N}}(d s, d u)\right\|\right)^{2} \\
& +9 \mathbf{E}\left(\left\|\zeta\left(\zeta I+\Gamma_{0}^{T}\right)^{-1} \sum_{0<t_{k}<t} \mathcal{T}\left(t-t_{k}\right) I_{k}\left(z_{a}\left(t_{k}\right)\right)\right\|\right)^{2} .
\end{aligned}
$$

By (H5), for all $0 \leq s \leq T$, the operator $\zeta\left(\zeta I+\Gamma_{0}^{T}\right)^{-1} \rightarrow 0$ strongly as $\zeta \rightarrow 0$; moreover, $\left\|\zeta\left(\zeta I+\Gamma_{0}^{T}\right)^{-1}\right\| \leq 1$. Thus, by the LDC theorem, we obtain $\mathbf{E}\left\|z_{\zeta}(T)-z_{T}\right\|^{2} \rightarrow 0$. Hence the proof. 


\section{Example}

Consider the stochastic control system:

$$
\begin{aligned}
& \begin{aligned}
d x(t, \theta)= & {\left[x_{\theta \theta}+B u(t, \theta)+\widetilde{f}(t, x(t))\right] d t+\widetilde{\sigma}(t, x(t)) d w(t) } \\
& +\int_{\mathcal{U}} \widetilde{h}(t, x(t), u) \widetilde{N}(d s, d u), \quad t \in J, t \neq t_{k},
\end{aligned} \\
& \begin{aligned}
\Delta x\left(t_{k}, \theta\right)=\widetilde{I}_{k}\left(x\left(t_{k}\right), \theta\right), \quad t=t_{k}, k=1,2, \ldots, m, \\
x(t, 0)=x(t, \pi)=0, \quad t \in[0, T], 0<\theta<\pi,
\end{aligned} \\
& x(0, \theta)+\sum_{i=1}^{n} \alpha_{i} x\left(t_{i}, \theta\right)=x_{0}(\theta), \quad \theta \in[0, \pi],
\end{aligned}
$$

where $B$ is a bounded linear operator from a Hilbert space $\mathrm{U}$ into $\mathrm{H} ; \tilde{f}: J \times \mathrm{H} \rightarrow \mathrm{H}, \widetilde{\sigma}: J \times$ $\mathrm{H} \rightarrow \mathcal{L}_{2}^{0}, \widetilde{h}: J \times \mathrm{X} \times \mathrm{U} \rightarrow \mathrm{H}$ are all continuous and uniformly bounded, $u(t)$ is a feedback control, and $w(t)$ is a $Q$-Wiener process.

Let $\mathrm{H}=\mathscr{L}_{2}[0, \pi]$, and let $A: D(A) \subset \mathrm{H} \rightarrow \mathrm{H}$ be an operator defined by $A x=x_{\theta \theta}$ with domain

$$
D(A)=\left\{x \in \mathrm{H}: x, x_{\theta} \text { are absolutely continuous } x_{\theta \theta} \in \mathrm{H}, x(0)=x(\pi)=0\right\} .
$$

Furthermore, $A$ has discrete spectrum, the eigenvalues are $-n^{2}, n=1,2, \ldots$, with the corresponding normalized characteristic vectors $e_{n}(s)=(2 / \pi)^{1 / 2} \sin n s$, then

$$
A x=\sum_{n=1}^{\infty}-n^{2}\left\langle x, e_{n}\right\rangle e_{n}, \quad x \in \mathrm{H} .
$$

Here, $A$ generates a compact semigroup $T(t), t>0$, in $\mathrm{H}$ and is given by

$$
T(t) x=\sum_{n=1}^{\infty} e^{-n^{2} t}\left\langle x, e_{n}\right\rangle e_{n}(\theta), \quad x \in \mathbb{H}
$$

Define $f:[0, T] \times \mathrm{H} \rightarrow \mathrm{H}, \sigma:[0, T] \times \mathrm{H} \rightarrow \mathcal{L}_{2}^{0}, h: J \times \mathrm{H} \times U \rightarrow \mathrm{H}$, and $I: \mathrm{H} \rightarrow \mathrm{H}$ by

$$
\begin{aligned}
& f(t, x(t))(\theta)=\widetilde{f}(t, x(t))(\theta), \\
& \sigma(t, x(t))(\theta)=\widetilde{\sigma}(t, x(t))(\theta), \\
& h(t, x(t), u)(\theta)=\widetilde{h}(t, x(t), u)(\theta), \\
& I_{k}\left(x\left(t_{k}\right)\right)(\theta)=\widetilde{I}_{k}\left(x\left(t_{k}\right)\right)(\theta), \quad(t, x(t)) \in[0, T], \theta \in[0, \pi] .
\end{aligned}
$$

The function $g: \mathscr{C}([0, T] ; \mathrm{H}) \rightarrow \mathrm{H}$ is defined as

$$
g(z)(\theta)=\sum_{i=1}^{n} \alpha_{i} x\left(t_{i}, \theta\right), \quad 0<t_{i}<T, \theta \in[0, \pi],
$$

with this choice of $A, B, f, \sigma, I$ and $g$, (1) is the abstract formulation of (6) such that the conditions in $(\mathrm{H} 1)-(\mathrm{H} 5)$ are satisfied. 
Now define an infinite-dimensional space

$$
\mathrm{U}=\left\{u: u=\sum_{n=2}^{\infty} u_{n} e_{n}(\theta) \mid \sum_{n=2}^{\infty} u_{n}^{2}<2\right\}
$$

with the norm defined by

$$
\|u\|_{\mathrm{U}}=\left(\sum_{n=2}^{\infty} u_{n}^{2}\right)^{\frac{1}{2}}
$$

and a linear continuous mapping $B$ from $\mathrm{U} \rightarrow \mathrm{H}$ as follows:

$$
B u=2 u_{2} e_{1}(\theta)+\sum_{n=2}^{\infty} u_{n}(t) e_{n}(\theta) .
$$

It is obvious that, for $u(t, \theta, \omega)=\sum_{n=2}^{\infty} u_{n}(t, \omega) e_{n}(\theta) \in \mathscr{L}_{2}^{\Im}([0, T] ; \mathrm{U})$,

$$
B u=2 u_{2}(t) e_{1}(\theta)+\sum_{n=2}^{\infty} u_{n}(t) e_{n}(\theta) \in \mathscr{L}_{2}^{\Im}([0, T] ; \mathrm{U})
$$

Moreover,

$$
\begin{aligned}
& B^{*} v=\left(2 v_{1}+v_{2}\right) e_{2}(\theta)+\sum_{n=3}^{\infty} v_{n} e_{n}(\theta) \\
& B^{*} S^{*}(t) x=\left(2 x_{1} e^{-t}+x_{2} e^{-4 t}\right) e_{2}(\theta)+\sum_{n=3}^{\infty} x_{n} e^{-n^{2} t} e_{n}(\theta)
\end{aligned}
$$

for $v=\sum_{n=1}^{\infty} v_{n} e_{n}(\theta)$ and $x=\sum_{n=1}^{\infty} x_{n} e_{n}(\theta)$. Let $\left\|B^{*} S^{*}(t) x\right\|=0, t \in[0, T]$, it follows that

$$
\begin{aligned}
& \left\|2 x_{1} e^{-t}+x_{2} e^{-4 t}\right\|^{2}+\sum_{n=3}^{\infty}\left\|x_{n} e^{-n^{2} t}\right\|^{2}=0, \quad t \in[0, T] \\
& \Rightarrow \quad 0, \quad n=1,2, \ldots \\
& \Rightarrow \quad x=0 .
\end{aligned}
$$

Thus, by Theorem 4.1.7 of [19], the deterministic linear system corresponding to (6) is approximate controllable on $[0, T]$. Therefore system (6) is approximate controllable provided that $f, \sigma, I$, and $g$ satisfy assumptions $(\mathrm{H} 1)-(\mathrm{H} 5)$.

\section{Conclusion}

In this paper we have established the approximate controllability of a semilinear impulsive stochastic system with nonlocal conditions and Poisson jumps in a Hilbert space. The results are obtained by using Sadovskii's fixed point theorem and semigroup theory. Further the results have been verified by a proper example. In future the criteria may be extended to semilinear impulsive stochastic integrodifferential equations driven by a fractional Brownian motion. 


\section{Acknowledgements}

The authors would like to thank the reviewers for their constructive comments in upgrading the article.

\section{Funding}

Not applicable.

\section{Availability of data and materials}

Not applicable.

\section{Competing interests}

The authors declare that they have no competing interests.

\section{Authors' contributions}

All authors read and approved the final manuscript.

\section{Author details}

${ }^{1}$ Department of Mathematics, PSG College of Arts and Science, Coimbatore, India. ${ }^{2}$ Department of Mathematics,

Cankaya University, Ankara, Turkey. ${ }^{3}$ Institute of Space Sciences, Magurele-Bucharest, Romania.

\section{Publisher's Note}

Springer Nature remains neutral with regard to jurisdictional claims in published maps and institutional affiliations.

Received: 13 February 2019 Accepted: 10 December 2019 Published online: 10 February 2020

\section{References}

1. Klamka, J.: Controllability of dynamical systems, a survey. Bull. Pol. Acad. Sci., Tech. Sci. 61, 221-229 (2013)

2. Anguraj, A., Ramkumar, K.: Approximate controllability of semilinear stochastic integrodifferential system with nonlocal conditions. Fractal Fract. 2(4), 29 (2018)

3. Sakthivel, R., Ganesh, R., Ren, Y., Anthoni, S.M.: Approximate controllability of nonlinear fractional dynamical systems. Commun. Nonlinear Sci. Numer. Simul. 18, 3498-3508 (2013)

4. Balachandran, K., Kim, J.H., Karthikeyan, S.: Controllability of semilinear stochastic integrodifferential equations. Kybernetika 43, 31-44 (2007)

5. Balachandran, K., Karthikeyan, S.: Controllability of stochastic integrodifferential systems. Int. J. Control 80, 486-491 (2007)

6. Klamka, J.: Stochastic controllability of linear systems with delay in control. Bull. Pol. Acad. Sci. 55, 23-29 (2007)

7. Ravichandran, C., Valliammal, N., Nieto, J.J.: New results on exact controllability of a class of fractional neutral integrodifferential systems with state-dependent delay in Banach spaces. J. Franklin Inst. 356(3), 1535-1565 (2019)

8. Mao, X.: Stochastic Differential Equations and Applications. Horwood, Chichester (1997)

9. Da Prato, G., Zabczyk, J.: Stochastic Equations in Infinite Dimensions. Encyclopedia of Mathematics and Its Applications, vol. 44. Cambridge University Press, Cambridge (1992)

10. Boudaoui, A., Lakhel, E.: Controllability of stochastic impulsive neutral functional differential equations driven by fractional Brownian motion with infinite delay. Differ. Equ. Dyn. Syst. 26(1-3), 247-263 (2018)

11. Sakthivel, R.: Approximate controllability of impulsive stochastic evolution equations. Funkc. Ekvacioj 52, 381-393 (2009)

12. Zhang, X., Agarwal, P., Liu, Z., Peng, H.: The general solution for impulsive differential equations with Riemann-Liouville fractional-order $q \in(1,2)$. Open Math. 13(1), 908-923 (2015)

13. Tariboon, J., Ntouyas, S.K., Agarwal, P.: New concepts of fractional quantum calculus and applications to impulsive fractional q-difference equations. Adv. Differ. Equ. 2015, 18 (2015)

14. Boufoussi, B., Hajji, S.: Successive approximation of neutral functional stochastic differential equations with jumps. Stat. Probab. Lett. 80, 324-332 (2010)

15. Zhao, $\mathrm{H}$.: On existence and uniqueness of stochastic evolution equation with Poisson jumps. Stat. Probab. Lett. 79(15), 2367-2373 (2009)

16. Sakthivel, R., Ren, Y.: Complete controllability of stochastic evolution equations with jumps. Rep. Math. Phys. 68, 163-174 (2011)

17. Luo, J., Liu, K.: Stability of infinite dimensional stochastic evolution equations with memory and Markovian jumps. Stoch. Process. Appl. 118, 864-895 (2008)

18. Mahmudov, N.I., Zorlu, S.: Controllability of nonlinear stochastic systems. J. Control 76, 95-104 (2003)

19. Kalman, R.E.: Controllability of linear systems. Contrib. Differ. Equ. 1, 190-213 (1963) 